\title{
A Converter of High Voltage Capacitor Charging Power Supply Using Piezoelectric Transformer
}

\author{
Chunyu Bai, Shiyi Li, Qiang Shen, Debang Cui \\ School of Aerospace Science and Engineering \\ Beijing Institute of Technology \\ Beijing, China \\ Email:sxbcy@126.com
}

\begin{abstract}
In order to adapt the miniaturization and low EMI of pulsed power supply, a novel Quasi-resonant piezoelectric transformer converter for high voltage pulse capacitor charging was developed. The system has the advantages of simple structure, absorbing the switch capacitive parameters and zero voltage switching (ZVS). Therefore, this converter is suit to operate under high-frequency conditions. The working principle of converter was introduced briefly, the working modes of inverter were analyzed and the formula calculating the resonant inductive and switch frequency was listed. At last, the design instance was described, the result of reality and calculated were well consistent.
\end{abstract}

Keywords- piezoelectric transformer; converter; Quasiresonant; quantum-mode control

\section{I . INTRODUCTION}

Piezoelectric Transformers (PTs), which transmits electrical energy through mechanical vibration, presents alternative characteristics to magnetic transformer. It is a very attractive device since it has many advantages, such as, potential low cost, small size and weight, no electromagnetic noise and high power density. PTs may become a viable alternative to magnetic transformers in various applications. However, there is little published report on PT applied to high voltage capacitor charging. Most of researches focus on applications to DC/DC converters and LCD backlighting [13]. And, those circuits given corresponding literature are too complicated to use in low-power PT applications.

In this paper, a study of a simple, single-ended quasiresonant amplifier is conducted. The proposed scheme adopts an external inductor which resonates with the PT input capacitance to make PT working high-efficiency. The output voltage is rectified and the resulting DC value is regulated by quantum-mode control strategy. In this way, the simplification of driving circuit and control circuit can be realized to most extent. The working modes of inverter were analyzed and the formula calculating the resonant inductive and switch frequency was listed. Finally, the design instance was described, the result of reality prove the proposed scheme to be available.

\section{II . CIRCUIT ARCHITECTURE}

Fig. 1 shows the single switch converter is composed of the switch $\mathrm{S}_{1}$, resonant inductor $\mathrm{L}_{\mathrm{R}}$, clamp diode $\mathrm{D}_{1}$, PT, voltage-double rectifier circuit, voltage divider, and the Project supported by State-level Key Laboratory Foundation of China (No.9140C360607060C36) control circuit. $\mathrm{C}_{1}$ is a high voltage load capacitor. $\mathrm{U}$ is $\mathrm{DC}$ input voltage source. $V_{S 1}$ is drain-source voltage of $S_{1}$, it is also input voltage of $\mathrm{PT}, \mathrm{V}_{\mathrm{GS} 1}$ is the gate signals of $\mathrm{S}_{1}, \mathrm{Hv}$ is output voltage. The dotted area shows an equivalent electrical circuit model of PT in proximity to the resonance frequency. The mechanical losses of the PT are modeled by $\mathrm{R}_{\mathrm{m}}$ and the resonant characteristics of the PT are modeled by $\mathrm{L}_{\mathrm{r}}$ and $\mathrm{C}_{\mathrm{r}}$. The capacitors $C_{i n}$ and $C_{o}$ represent the clamped input and output capacitance of the PT [3].

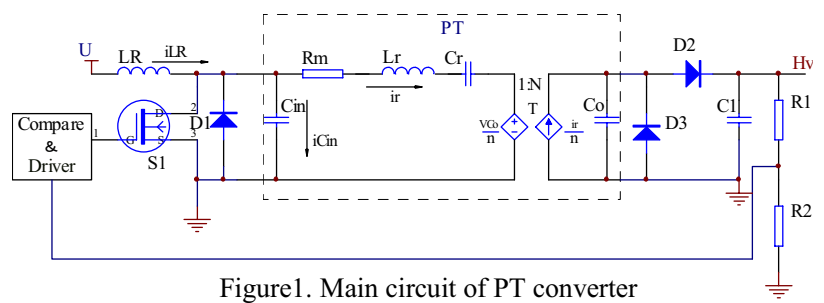

The switch $\mathrm{S}_{1}$ switches one time completes for one cycle. When $S_{1}$ is turned on, the inductor is charged. When $S_{1}$ is turned off, the energy stored in the inductor is transferred to the PT input capacitance, thus producing a resonant voltage. Through PT transformation and rectifying by rectifier circuit, a pulsation direct current will be produced to charge the highvoltage capacitor. The control circuit accepts the output voltage feedback signal which the voltage-division resistance gives, compares with the preinstall voltage value, and controls switch $\mathrm{S}_{1}$ action.

Based on the switch state, the converter operation can be broken into two main models of operation discussed as follows:



(a) $\mathrm{T}_{\text {on }}$

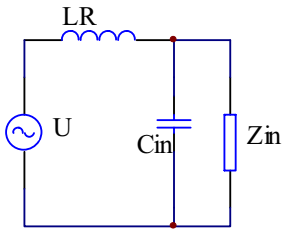

(b) $\mathrm{T}_{\text {off }}$
Figure2. Equivalent circuits of two modes

Mode $1 t \in\left[t_{0}, t_{1}\right]$ : At $t=t_{0}$. The switch $\mathrm{S}_{1}$ is turned on. Because static drain-to-source on-resistance of the switch $S_{1}$ is very low, the input terminal of PT is shorted. A current flow into the stage and the inductor is charged. The equivalent circuit is shown in Fig.2 (a).Neglecting the inductance equivalent series resistance and the switch $\mathrm{S}_{1}$ on-resistance, the circuit satisfies: 


$$
L_{R} \frac{d i_{L R}}{d t}=U .
$$

The resonant current can be obtained from above equation.

$$
i_{L R}(t)=\frac{U}{L_{R}}\left(t-t_{0}\right)+i_{L R}\left(t_{0}\right)
$$

Mode $2 t \in\left[t_{1}, t_{2}\right]:$ At $t=t_{1}$. The switch $\mathrm{S}_{1}$ is turned off, the stored magnetic energy is discharged to $\mathrm{C}_{\text {in }}$ by the resonance with $L_{R}$ and $V_{S 1}$ becomes the half sine waveforms. The operational frequency is chosen to be greater than the resonant frequency so that the input impedance must be inductive, which guarantee the switch achieves ZVS. Because the input capacitance $\mathrm{C}_{\text {in }}$ provides a low impedance path compared to the mechanical branch of the PT, the major resonant component is $\mathrm{C}_{\mathrm{in}}$ for the inverter, $i_{C i n} \gg i_{L r}$. The equivalent circuit is shown in Fig.2 (b).

$$
\begin{aligned}
& L_{R} \frac{d i_{L R}}{d t}+u_{C i n}=U \\
& C_{i n} \frac{d u_{C i n}}{d t}=i_{C i n} \\
& i_{L R}=i_{C i n}
\end{aligned}
$$

From (3), (4) and (5) we can obtain input voltage of PT and resonant current:

$$
\begin{aligned}
& u_{C i n}(t)=Z i_{L R}\left(t_{1}\right) \sin \omega_{0}\left(t-t_{1}\right)+\left[1-\cos \omega_{0}\left(t-t_{1}\right)\right] U \\
& i_{L R}(t)=\frac{U}{Z} \sin \omega_{0}\left(t-t_{1}\right)+i_{L R}\left(t_{1}\right) \cos \omega_{0}\left(t-t_{1}\right) \\
& \text { here, } \quad \omega_{0}=\frac{1}{\sqrt{L_{R} C_{i n}}}, Z=\sqrt{\frac{L_{R}}{C_{i n}}} .
\end{aligned}
$$

At $t=t_{2}$, the switch $\mathrm{S}_{1}$ is turn on and next period is beginning. We can find that peak value of the switch $S_{1}$ voltage changes along with the value of duty factor in given PT and resonant inductance situation from (2) and (6). In order to reduce the switch voltage stress, the value of duty factor cannot be too big. It should be determinate after being overall trade-off among PT step-up ratio, the output voltage request and drain-to-source breakdown voltage of the switch.

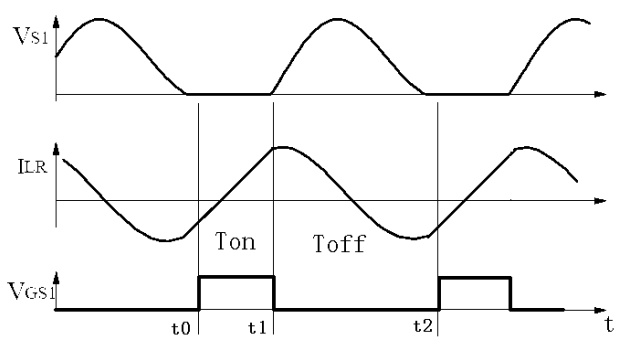

Figure3. Key waveform of PT inverter

Fig. 3 shows the key waveform of the PT inverter, when the inverter circuit works at a fixed frequency and fixed duty factor. Obviously the switch $S_{1}$ works in zero voltage on-off state.

\section{CONTROL STRATEGY}

In order to stabilize output voltage within the expectant range, quantum-mode control strategy was adopted. The operation principle of quantum-mode control is very simple [4]. The inverter is switched with constant-frequency and constant-duty cycle, so that a constant square voltage is applied to the series inductor and the PT. This way, the converter output voltage which eventually reaches the maximum allowable value rises gradually. This situation is detected by the control circuit that stops driving the switches of the inverter. In this time, the PT input is null and the converter output voltage decreases. When the minimum allowable value for the output voltage is reached, the control circuit activates the inverter switches again and the cycle starts once more.

The most important feature of this kind of control is not necessary to perform a dynamic analysis of the power stage. All that needs to be done is to make sure that the PT is safely driven in terms of maximum input voltage. This simplifies the design of the control circuitry. This circuit has several advantages: simplicity, size, and price, plus an inherent noload protection. This is important, because no-load condition is dangerous for the PT, since this situation would place energy at its input, but no energy would be drawn from its output, which means all the energy would be dissipated in the PT itself. Too much temperature rise will make PT broken. The operation mode selected provides shutdown under no-load condition, thus avoiding this critical situation. As far as shortcircuit protection is concerned, the PT is self-protected against this condition, for its impedance increases when a short-circuit load is connected across its secondary terminals, thus limiting current flow through the device.

\section{IV.SELECTION AND CALCULATION OF MAIN PARAMETERS}

\section{A. Switch Frequency Selection}

For a given load, the selected switch frequency should make PT work at the maximum gain state. In the topology circuit concerned in this paper, PT load is a voltage-double rectifier circuit containing high voltage capacitor. As shown in Fig 4(a), the PT equivalent load changes according to the variations of charging voltage in the process of capacitor charging. It also shows that the optimum switch frequency is not a fixed value but in a frequencies interval. So, it needs to select a relatively optimal switch frequency when using quantum mode control converters. To simplify the analysis, the PT equivalent circuit and load are transformed equivalently as below:

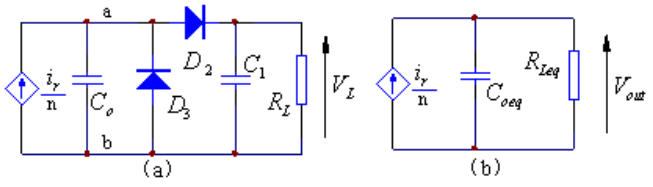

Figure4. Topology of a voltage-double rectifier and It's equivalent circuit fed by the current (ir/n) 
The output of voltage-double rectifier circuit and its load can be equivalent to parallel resistance and capacitance network[5], as shown in Fig. 4(b). Fig. 5(b) shows the equivalent circuit obtained after reflecting the output part of the equivalent circuit to the input side. The parallel network can be transformed to a serial frequency dependent network for further simplified analysis, as shown in Fig. 5(c):



Figure5. Equivalent circuit of the system: PT, output rectifier and load

The relations between $C_{\text {oeq }}$, load resistance $R_{\text {Leq }}$, output voltage $V_{\text {out }}$ and corresponding $C_{o}^{\prime}$, reflecting resistance $R_{o}^{\prime}$, reflecting voltage $V_{\text {out }}^{\prime}$ are respectively shown below:

$$
\begin{aligned}
R_{o}^{\prime} & =\frac{R_{\text {Leq }}}{N^{2}} \\
C_{o}^{\prime} & =N^{2} C_{\text {oeq }} \\
V_{\text {out }}^{\prime} & =\frac{V_{\text {out }}}{N}
\end{aligned}
$$

The serial circuit made up of $C_{o}^{\prime \prime}$ and $R_{o}^{\prime \prime}$ is equivalent to the parallel network containing $C_{o e q}$ and $R_{L e q}$, then:

$$
\begin{aligned}
& R_{o}^{\prime \prime}=\frac{R_{\text {Leq }}}{N^{2}\left(1+C_{o e q}^{2} R_{L e q}^{2} \omega^{2}\right)} \\
& C_{o}^{\prime \prime}=\frac{N^{2}\left(1+C_{o e q}^{2} R_{L e q}^{2} \omega^{2}\right)}{C_{o e q} R_{o e q}^{2} \omega^{2}}
\end{aligned}
$$

When the input voltage $V_{\text {in }}$ and the current $i_{r}$ which flows into the resonant branch circuit have the same phase, PT has the maximum voltage gain [3]. Based on the equivalent circuit model in Fig. 5(c), transfer function can be deducted to:

$$
\frac{i_{r}}{V_{i n}}(j \omega)=\frac{j \omega C_{e q}(\omega)}{1-L_{r} C_{e q}(\omega) \omega^{2}+j \omega R_{e q}(\omega) C_{e q}(\omega)} .
$$

Where:

$$
\begin{aligned}
& R_{e q}(\omega)=R_{m}+R_{o}^{\prime \prime}(\omega) \\
& C_{e q}(\omega)=\frac{C_{r} C_{o}^{\prime \prime}(\omega)}{C_{r}+C_{o}^{\prime \prime}(\omega)}
\end{aligned}
$$

Obviously for (13) the maximum voltage gain is obtained when it satisfies the condition below:

$$
1-L_{r} C_{e q}\left(\omega_{o p t}\right) \omega_{o p t}^{2}=0
$$

Applying (12), (15), and (16):

$$
\begin{aligned}
& \left(C_{r} L_{r} C_{o e q}^{2} R_{\text {Leq }}^{2} N^{2}\right) \omega_{\text {opt }}^{4}- \\
& \left(C_{r} C_{o e q} R_{\text {Leq }}^{2}+C_{o e q}^{2} R_{\text {Leq }}^{2} N^{2}-C_{r} L_{r} N^{2}\right) \omega_{o p t}^{2}=N^{2}
\end{aligned}
$$

In the initial stage of high voltage capacitor charging, the charging process has a good constant current characteristic. The voltage of capacitor at this stage is relatively low, which can be approximated as short-circuit, then the corresponding optimization frequency can be obtained from (17).

$$
f_{\text {opt } 1}=\frac{1}{2 \pi \sqrt{L_{r} C_{r}}}
$$

When it gets to the end of the capacitor charging, the terminal voltage of capacitor is increasing accompanied with the currents at every action period showing the trend of current attenuation. A relatively high voltage gain of PT can restrain this kind of current attenuation to get a better charging efficiency. So in the quantum control mode, the selection of inverter circuit switch frequency should make the whole circuit get a maximum voltage gain at the end of charging, which can realize the constant current charging characteristic in the whole charging process. Meanwhile, the switch frequency is greater than the mechanical resonance frequency, which makes it convenient to realize zero-voltage-switching, and because the selected frequency value is deviated from that in (18), it is also effective to restrain the starting current in charging process.

The equivalent resistance and capacitance of voltagedouble rectifier circuit in the end of charging satisfy:

$$
\begin{aligned}
& R_{\text {Leq }}=\frac{R_{L}}{8}, \\
& C_{\text {oeq }}=C_{o} .
\end{aligned}
$$

Applying above equation to (17), the selected switch frequency can be got:

$$
f_{S}=\frac{\sqrt{C_{r} C_{o} R_{L}^{2}+C_{o}^{2} R_{L}^{2} N^{2}-64 C_{r} L_{r} N^{2}+\sqrt{256 C_{r} L_{r} C_{o}^{2} R_{L}^{2} N^{4}+\left(C_{r} C_{o} R_{L}^{2}+C_{o}^{2} R_{L}^{2} N^{2}-64 C_{r} L_{r} N^{2}\right)^{2}}}}{\pi C_{o} R_{L} N \sqrt{8 C_{r} L_{r}}}
$$

\section{B. Calculation of Inductance Parameter}

As shown in Fig. 1, inductor $L_{R}$ not only plays a role of low-pass filter together with the input capacitance $\mathrm{C}_{\text {in }}$ of PT, but also is an effective approach to get the soft-switching mode. If the taken value of $\mathrm{L}_{\mathrm{R}}$ is too small, it will produce non-sinusoidal oscillatory wave and the circuit working current will increase, which make the efficiency of the circuit reduced; if the taken value of $L_{R}$ is too big, it will decrease the 
voltage applied to the piezoelectric transformer, which demands the circuit to have a larger gain.

A method is proposed in [6], the inductor's value must be carefully selected so that the electrical resonance frequency with the input capacitance is as close as possible to the mechanical resonance frequency of PT, which satisfies the relation below:

$$
f_{r}=\frac{1}{2 \pi \sqrt{L_{R} C_{i n}}}
$$

But for $\mathrm{V}_{\mathrm{GS}}$ signals with any duty cycle, $\mathrm{L}_{\mathrm{R}}$ calculated by above method can't realize zero-voltage switch operations very well. The basic condition of ZVS realization is taken into account in combination with (6), then $L_{R}$ is selected to make the inverter circuit satisfy that $\mathrm{U}_{\text {cin }}$ can cross zero at both beginning and end of the off-time $\mathrm{V}_{\mathrm{GS}}$. At shown in the relation below:

$$
\left[\pi+2 \arctan \frac{U}{Z i_{L R}\left(t_{1}\right)}\right] \sqrt{L_{R} C_{i n}}=\frac{1}{f_{S}}(1-D)
$$

Where, $f_{s}$ is the switch frequency and $D$ is the duty factor of switch frequency signal.

Given input signal with a certain duty factor, an improved method is proposed in this paper. $L_{R}$ 's initial value should be calculated by (21) at first, then it is modified by (22) to determine the concrete value of inductor $L_{R}$ after the frequency and duty cycle of the switch signal are determined.

\section{EXPERIMENTAL RESULTS}

In order to validate the proposed topology and control method, an experimental prototype is developed by using KHMPT2207 piezoelectric transformer. The main parameters are listed below: $\mathrm{L}_{\mathrm{r}}=8.1 \mathrm{mH}, \mathrm{C}_{\mathrm{r}}=510 \mathrm{pF}, \mathrm{R}_{\mathrm{m}}=7.3 \Omega, \mathrm{C}_{\mathrm{in}}=78 \mathrm{nf}$, $\mathrm{C}_{\mathrm{o}}=100 \mathrm{pf}, \mathrm{n}=55$. Switch uses power MOSFET, load high voltage capacitor is $0.15 \mathrm{uf} / 3.5 \mathrm{KV}$. When input dc voltage is $28 \mathrm{~V}$, switch frequency $\mathrm{f}_{\mathrm{s}}$ is selected as $79 \mathrm{KHz}$ and duty factor is 0.2 , inductance calculated by the method above is $68 \mathrm{uH}$. Experimental waveforms of switch input voltage $\mathrm{V}_{\mathrm{GS}}$ and $\mathrm{PT}$ input voltage $\mathrm{V}_{\mathrm{S} 1}$ are shown in Fig.6. It also shows that the inverter switch of the actual circuit works in the zero-voltage soft switch mode. The maximum value of $\mathrm{V}_{\mathrm{S} 1}$ is $70 \mathrm{~V}$, which is consistent to the result of (6).

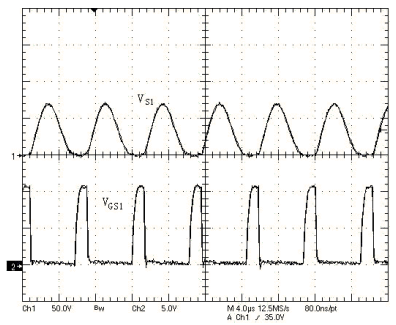

Figure6. Experimental waveforms of $\mathrm{V}_{\mathrm{GS} 1}$ and $\mathrm{V}_{\mathrm{S} 1}$

Fig. 7 shows the waveforms of PT input voltage $\mathrm{V}_{\mathrm{S} 1}$ and load capacitor output voltage $\mathrm{V}_{\mathrm{C} 1}$ which ranges from $2.7 \mathrm{KV}$ to
$2.9 \mathrm{KV}$. It also shows the variation process of output voltage under quantum control mode. The rising linearly section of $\mathrm{V}_{\mathrm{C} 1}$ curve tells us that this circuit has realized constant current charging with high efficiency.

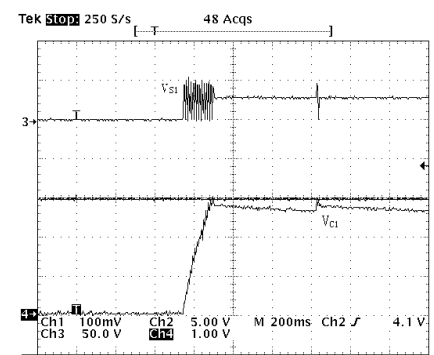

Figure7. Experimental waveforms of $\mathrm{V}_{\mathrm{S} 1}$ and $\mathrm{V}_{\mathrm{C} 1}$

\section{CONCLUSIONS}

A topology suitable to drive PT in high voltage capacitor charging power supply converters has been presented. The main advantages of this topology are its simplicity and the low number of additional components needed: only one extra inductor needs to be included. Meanwhile this topology can maintain constant current changing for high voltage capacitor and this proposed circuit is able to have its switches achieve ZVS in order to have very low switching losses. Tests allowed verifying that, in the case of short-circuit in the load; the piezoelectric transformer behaves in such a way that the converter is self-protected. The experimental results prove the proposed scheme to be available.

\section{ACKNOWLEDGMENT}

The authors are grateful to KangHong Corp. for providing us with the piezoelectric transformer, without which it would have been impossible to develop the presented device, and to colleagues in State Key Laboratory, Xi Ren and Shoubao Li for valuable suggestion and fruitful discussions.

\section{REFERENCES}

[1] Yu-Kang Lo, and Kai-Jun Pai, "Feedback design of a piezoelectric transformer-based half-Bridge resonant CCFL inverter," IEEE Transactions on Industrial Electronics. Vol. 54, pp.2716-2723, Oct. 2007

[2] Dallago E, and Danioni A, "Resonance frequency tracking control for piezoelectric transformer DC-DC converter," Electron Lett. Vol. 37, pp.1317-1318, Oct 2001

[3] Gregory Ivensky, Isaac Zafrany, and Sam Ben-Yaakov, "Generic Operational Characteristics of Piezoelectric Transformers," IEEE Transactions on Power Electronics. Vol. 17, pp.1049-1057 .Nov. 2002.

[4] Nuno F, Martin J.A, and Diaz. J, "Quantum mode control for piezoelectric transformers in AC/DC applications," Power Electronics Congress, 2002. Technical Proceedings. pp.202-207, Oct. 2002.

[5] Ivensky G, Shvartsas M, and Ben-Yaakov S. "Analysis and modeling of a voltage doubler rectifier fed by a piezoelectric transformer," IEEE Transactions on Power Electronics. Vol. 19, pp.542-549, March 2004.

[6] Dallago E, Danioni A, and Ricotti G, "Single chip, low supply voltage piezoelectric transformer controller," European Solid-State Circuits.2003 ESSCIRC '03 Conference.pp.273-276, Sept. 2003. 